\title{
Constant Proportion Portfolio Insurance Strategy in Southeast European Markets
}

\author{
Elma Agić-Šabeta \\ Bosna Bank International, Bosnia and Herzegovina
}

\section{Abstract}

Background: In today's highly volatile and unpredictable market conditions, there are very few investment strategies that may offer a certain form of capital protection. The concept of portfolio insurance strategies presents an attractive investment opportunity. Objectives: The main objective of this article is to test the use of portfolio insurance strategies in Southeast European (SEE) markets. A special attention is given to modelling non-risky assets of the portfolio. Methods/Approach: Monte Carlo simulations are used to test the buy-and-hold, the constant-mix, and the constant proportion portfolio insurance (CPPI) investment strategies. A covariance discretization method is used for parameter estimation of bond returns. Results: According to the risk-adjusted return, a conservative constant mix was the best, the buy-and-hold was the second-best, and the CPPI the worst strategy in bull markets. In bear markets, the CPPI was the best in a high-volatility scenario, whereas the buy-and-hold had the same results in low- and medium-volatility conditions. In no-trend markets, the buy-and-hold was the first, the constant mix the second, and the CPPI the worst strategy. Higher transaction costs in SEE influence the efficiency of the CPPI strategy. Conclusions: Implementing the CPPI strategy in SEE could be done by combining stock markets from the region with government bond markets from Germany due to a lack of liquidity of the government bond market in SEE.

Keywords: investments; portfolio insurance; constant proportion portfolio insurance; Monte Carlo simulations; interest rate models

JEL classification: C15, G12

Paper type: Research article

Received: Jul 24, 2015

Accepted: Sep 13, 2015

Citation: Agić-Šabeta, E. (2016), "Constant Proportion Portfolio Insurance Strategy in Southeast European Markets", Business Systems Research, Vol. 7, No. 1, pp. 59-80.

DOI: 10.1515/bsrj-2016-0005

\section{Introduction}

Bachelier (1900) discovered that price changes in financial markets followed a normal distribution. Therefore, it was possible to present stock prices on a relatively narrow bell curve (Bachelier 1900). However, financial theorists after him found that extreme events can cause significant deviations from the normal curves 
(Mandelbrot 1963, Blattberg et al. 1974, Patton 2004, Bouchard 2008). In statistics these deviations are called outliers. In finance they are known as fat tails.

Events such as a fall of the S\&P 500 index by $23 \%$ on October $19^{\text {th }} 1987$ or a dramatic increase of the NASDAQ composite index by more than $14 \%$ on January 3 rd 2001 were major surprises according to accepted financial theory. The probability of such events is very low, but it exists. For instance, the case of the S\&P 500 drop mentioned would have had nineteen standard deviations from the mean (Mauboussin et al. 2002). Hence, making financial decisions based on the assumption of normal distributions can be very dangerous. The question is whether there is any way to counteract such risks. This paper tests strategies that may be applied to counteract such events.

Specific risks can be reduced through diversification. Market risks that cannot be related to a single company, but are external and have an impact on the entire economy, cannot be reduced through diversification. Portfolio insurance strategies play a significant role in reducing market risks (Steiner et al. 2002).

Financial markets in Southeast Europe (SEE) may be described as emerging markets where risks and volatilities are higher compared to developed financial markets. Investment strategies that offer capital protection are rarely or never used in SEE markets, and therefore this market was selected for testing in this article.

The purpose of this study is to test portfolio insurance strategies in SEE. The research contribution of this article is following. First, the basic concept of portfolio insurance investment strategies is presented. The constant proportion portfolio insurance (CPPI) investment strategy is tested in SEE markets. Second, advanced risk modelling techniques have been applied, which have not been used in academic research and financial practice in the region. The main steps of the modelling approach and parameter estimation are described. Third, a stochastic interest rate model to simulate non-risky asset return has been used, which is a more realistic approach, as opposed to published research in this field so far.

The structure of the paper is as follows. The introduction offers background information. The literature overview presents the theoretical concept and recent research on the topic. The methodology section describes the steps in testing the investment strategies. The results section presents the evaluation of strategies. The conclusion includes a discussion, practical recommendations, and limitations of the research.

\section{Literature Review}

\section{Portfolio Insurance}

Academics and practitioners have developed strategies that make it possible to face the unpredictable market environment with greater confidence. Various strategies are being put into place in order to reduce market risks. Leland and Rubinstein (1976), invented the concept of portfolio insurance, which aims at providing capital protection and at the same time allowing participation in upside market movements (Laurent 2003).

Pension funds withdrew their funds in stocks due to the 1973-1974 stock market declines. Therefore, Leland wanted to develop some form of insurance that would make it possible to keep such funds in a portfolio even during a time of market decline. Although he was trying to understand the option pricing formula by Black and Scholes, he realized that dynamic hedging of options with stocks could be achieved by the reverse process in which a stock is hedged with a risk-free asset (Leland et al. 1988). 
Portfolio insurance strategies, limit the undesired loss, but the possible profit is decreased by the amount of option premium that has to be paid (Kolb 2003). This is reflecting how investors see risk: they consider risky returns below some certain value (Laurent 2003). By using portfolio insurance strategies, the conversion of a symmetric into an asymmetric performance profile takes place, as shown in Figurel (MeyerBullerdiek 2004).

Figure 1

Asymmetric performance profile of investors

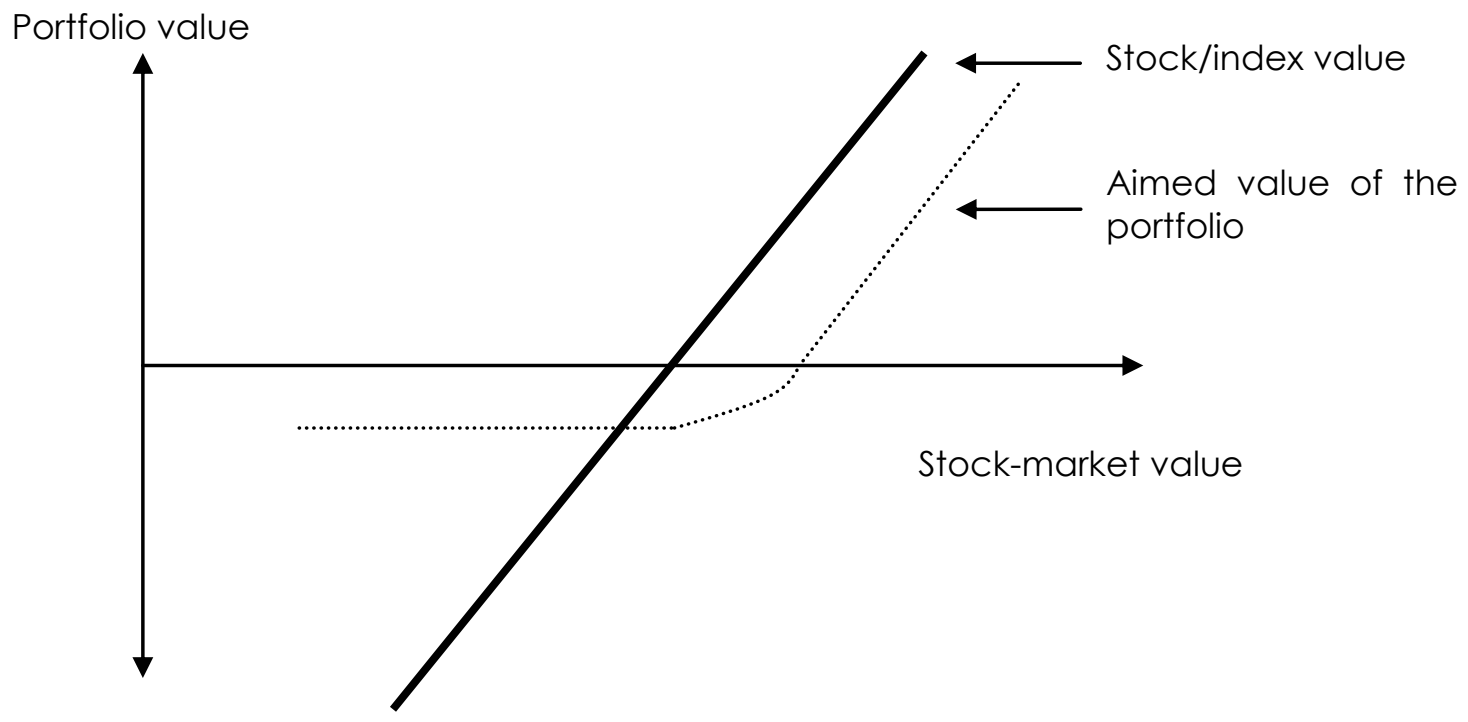

Source: Meyer-Bullerdiek 2004, p.31

There is wide range of portfolio insurance strategies and they can be subdivided into static and dynamic ones. Furthermore, portfolio insurance strategies can be divided into option-based strategies, option-duplicating strategies, and derivative independent strategies or spot strategies. The details of executing portfolio insurance strategies are described in Rudolf (1995), Bodie et al.(2005), Hull (2003), Kosowski et al. (2015), and elsewhere. Currently, the two most popular dynamic strategies using portfolio insurance are the synthetic protective put approach by Rubinstein and Leland (1981) and CPPI by Black and Jones (1987) and Black and Perold (1992) (Bassak 2002).

The focus of this paper is on CPPI strategy in SEE markets, which could be carried out because its execution is not based on option-pricing theory. In SEE, usage of derivatives is limited. Derivatives are primarily used by banks to hedge their interest and currency risks (Croatian Central Bank 2010). In Bosnia, one bank only reported some kind of derivative contracts in 2010 (Banking Agency of the Federation of Bosnia and Herzegovina, 2010). Therefore, derivative-free porffolio insurance strategies present an attractive opportunity for investors.

\section{Buy-and-hold, constant Mix and CPPI}

In order to evaluate various investment strategies, the buy-and-hold, constant-mix, and CPPI investment strategies are defined below.

The buy-and-hold investment strategy allocates its assets at the beginning of the investment horizon and holds the portfolio to the end, making no adjustments. The 
dividends received during the holding period are reinvested in the same asset at prevailing market rates.

The constant-mix strategy is a dynamic derivative-free strategy. It allocates assets based on a preset investment policy, whereby the shares of specific asset classes are identical at the beginning of each period (Zhao 2000). This means that the rebalancing is done when relative changes in asset classes' proportions occur at the preset dates. For example, such dates could be yearly, quarterly, or monthly. For instance, $70 \%$ of the portfolio is comprised of bonds and $30 \%$ stocks. This strategy is also described as a concave investment strategy that performs well in times of market reversals (Perold et al. 1995). In times when markets are continuous, either rising or falling, this investment strategy yields poor results as opposed to the buyand-hold and CPPI strategies.

The CPPI investment strategy invented by Black and Jones (1987) and Black and Perold (1992) is a dynamic portfolio insurance strategy without using derivatives. In principle, the CPPI strategy is a trading rule in which dynamic rebalancing of assets is implemented based on market conditions. It may also be described as a procyclical strategy that increases the share of risky assets in times of rising market value of a risky asset and, vice versa, decreases the share of risky assets in times of falling market values (Bertsch et al. 2002). The amount of risky asset in a portfolio is determined by an algorithm:

Figure 2

CPPI Algorithm

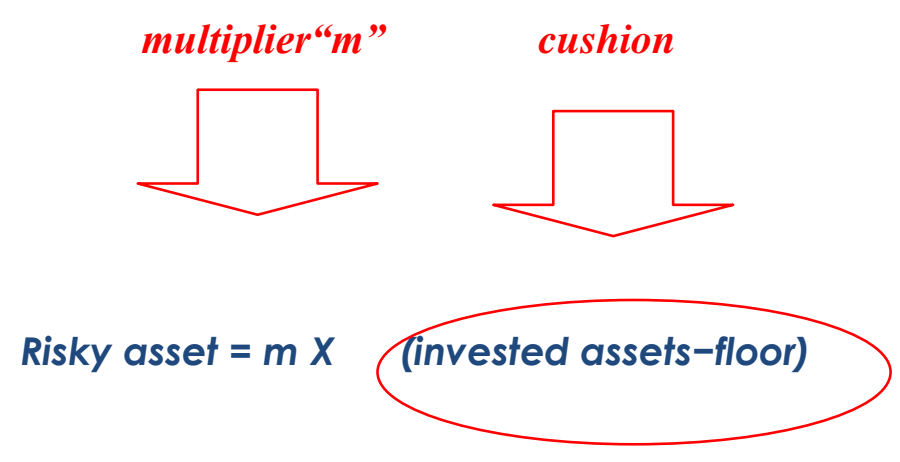

Source: Author's illustration

The floor represents the part of invested capital that is supposed to be protected by the end of the investment horizon. The difference between the current portfolio value and the floor is called the cushion. The cushion is multiplied by the multiplier $\mathrm{m}$, which is the amount invested in the risky portfolio component. Both the floor and the multiplier value are determined by the investor (Rudolf 1994).

The multiplier is always higher than 1, and typically ranges from 2 to 7 . The floor value grows at the amount of risk-free interest rate and is smaller than total invested assets. The higher the multiplier, the higher the share of risky assets is. However, the higher the multiplier, the faster the portfolio will reach the floor value in times of continuously falling markets (Perold et al. 1995).

Cesari and Cremonini (2003) test the performance of the CPPI strategy and conclude that CPPI strategies dominate in bear and no-trend markets, whereas constant-mix strategies dominate in bull markets according to risk-adjusted 
performance measures. Pain and Rand (2008) investigate the latest developments in portfolio insurance and explore the link between portfolio insurance and its implications on financial stability. They found that caution is needed in times of instable market conditions, when portfolio insurance may amplify financial instability. Constantinou et al. (2008) investigate statistical properties in the implementation of CPPI. They conclude that a good multiplier value for CPPI ranges from 3 to 5, and that appropriate rebalancing frequency is monthly. Furthermore, they conclude that an increased jump size and frequency of price changes of a risky asset have a positive impact on the return of CPPI strategy. Dichtl and Drobetz (2010) study CPPI from the behavioural science point of view. They discovered that the popularity of CPPI may be explained from the aspect of Tversky and Khaneman's (1992) cumulative prospect theory. This is due to the fact that, despite a lower return potential, a higher protection level is more desirable for investors from the view of prospective theory. Schöttle and Werner (2010) use mean-variance optimization in order to create a portfolio that represents a risky asset. They prove that CPPI strategy is more successful if the risky asset comprises an optimized portfolio of assets.

Portfolio insurance investment strategies have been more dominant in Europe than in the United States. The main investors in portfolio insurance products are highnet-worth individuals, private banks that buy products in order to sell them to their clients, and pension funds (Pain et al. 2008). Currently, CPPI-based products usually include options (Kosowski et al. 2015). CPPI and structured products based on CPPI have been offered in Asia (Wozniak 2006). The first investment fund based on CPPI was founded in Pakistan in 2012 (www.ublfunds.pk).

The main aim of this paper is to shed light on the attractiveness of portfolio insurance strategies and test the CPPI in SEE markets. The results are compared with similar studies in developed financial markets. In addition, the use of interest rate simulations is used to test the strategy, where the solutions for estimating parameter values are offered.

\section{Methodology}

Numerous articles have been written investigating the efficiency of the CPPI investment strategy (e.g., Cesari and Cremonini 2003, Constantinou et al. 2008, Dersch 2010, Dichtl and Drobetz 2010). Various types of models are used to simulate risky assets in a portfolio. Many of them use Monte Carlo simulations to simulate stock returns based on normal distribution of returns (e.g., Pain and Rand 2010, Dichtl and Drobetz 2010). However, no paper has been identified focusing on the simulation of a non-risky asset of the portfolio. Pain and Rand (2008) warn that interest rate movements may have a significant impact on the portfolio value of the CPPI investment strategy due to the interest rate impact on the value of a non-risky asset. However, they use a constant rate of 3\% for treasury bills for testing the strategies.

Dersch (2010) tests the CPPI strategy using historical data for the risky component of the portfolio. Dichtl and Drobetz (2010) use theoretical assumptions in bootstrap and Monte Carlo simulations. Schöttle and Werner (2010) use mean-variance optimization in order to create a portfolio that represents a risky asset. Cesari and Cremonini (2003) use theoretical ranges for average returns and standard deviations. Constantinou et al. (2008) use Student's distribution instead of normal distribution for Brownian motion of stock prices. In addition, they test the jump diffusion effect on stock prices. They use a constant $5 \%$ interest rate for bond returns.

This paper uses Monte Carlo simulations and normal distribution of return for stock returns and Cox-Ingersoll-Ross model for bond returns. 


\section{Monte Carlo simulations}

The well-known JP Morgan value at risk model is calculated by using historical prices and Monte Carlo simulations (Cottrell et al. 1996). Advanced financial institutions today use Monte Carlo simulations for risk modeling and stress testing purposes, as well as derivatives pricing. Based on interviews with financial institutions in Croatia, Serbia, and Bosnia and Herzegovina, none of them use Monte Carlo risk indicators, but their parent institutions abroad calculate these benchmarks.

The core idea of Monte Carlo simulation is generating random walk variables. Random walk is a frequently used mathematical model and serves as a basis for many stochastic processes. Most option pricing models, including the Black-Scholes model and many methods of modern risk management, assume that stock prices follow a random walk. Although the name of this simulation comes from the town in Monaco, the number of simulations that can be generated by a Monte Carlo simulation could not be produced there even if all the casinos worked without interruption for a million years (Deutsch 1998).

Monte Carlo simulations may be implemented using MS Excel but, if the volume of data is too large (e.g., for many stocks in a portfolio), simulations may be implemented in matlab, $\mathrm{C}_{++}$, or Java. The testing results have a high confidence level and it is very simple to change input values and create various scenarios. For more details on Monte Carlo simulations Glassermann (2004) should be considered.

The typical steps of Monte Carlo simulations are described in detail below.

Step 1.Selection of assets: For the needs of testing long-term investment strategies, ideally the daily, weekly, or monthly data on total return should be taken from a period of ten to fifteen years.

Risky asset: The Sarajevo Stock Exchange index SASX 10 was chosen as a representative of the risky asset. An important characteristic of the CPPI strategy is the need for liquidity of the investment vehicle. The SASX 10 index comprises ten stocks with the highest market capitalization and liquidity (www.sase.ba). Additional proof is the empirical research by Zaimovic (2010). Based on the number and turnover of stocks traded, she found twenty-seven different stocks that had the highest liquidity between 2005 and 2009. All stocks from the SASX 10 index can be found in these twenty-seven stocks. This index was introduced in 2006, which is a long time series, but still not able to create all the necessary scenarios for testing purposes. Thus, the price of gold expressed in EUR was added to the testing sample in order to create additional testing scenarios.

In both indices, the volatility over a long-term period has never been low, and therefore a hypothetical volatility was assumed for the low-volatility scenario in all markets. High volatility was also a hypothetical assumption in no-trend and bear markets.

The SASX 10 is a price index and does not include dividends. Usually, for the needs of testing, a total return index that includes both price changes and dividends should be used. The only equity index in regional markets that includes dividends is CROBEXtr, which was introduced in the Zagreb Stock Exchange in 2012 (www.zse.hr). This time series is too short for data calibration in this paper.

Non-risky asset: Although volumes of issued government treasury bills and bonds in regional financial markets are relatively high, these bonds are mostly held to maturity and trading with them is rare. There is a bond market index in the Zagreb Stock Exchange, but it was introduced recently. Therefore, the non-risky asset in this study was taken from the German bond market in order to satisfy the criteria of non-risky 
financial instrument in the CPPI strategy and to avoid foreign exchange differences following the suggestions by Damodoran (2008).

Usually, the non-risky asset in the CPPI strategy is represented by treasury bills. However, the German bond market is highly liquid and the long-term generic government bond index with a maturity of ten years (GDBR 10, available through Bloomberg) was chosen in this study. It was assumed that the coupon payments throughout the investment period would not have a significant impact on the performance of investment strategies.

Step 2: Selection of a stochastic model will be described for both risky and non-risky asset.

Risky asset: The process in which the present value only has an impact on future, but not the historical path itself, is named the Markov process. The assumption is that the present value of a risky variable-for instance, the stock price-fully reflects the historical information as in the frame of a weak form of an efficient market theory. Nevertheless, the future stock price depends only on the present value and external factors, such as politics, but not on stock prices in the past (Deutsch 1998).

Consequently, the development of the model is based on the deterministic variable named drift and a random variable. The random variable can take two directions only: $2 d$, and the only condition for its change is the passing of time $d t$. During the entire random walk, from $t$ (today) until $T$ (date in the future), time passes:

$$
T-t=n d t
$$

Because $d t$ is constant, the number of steps is equal to the time elapsed, $T-t$. The evolution of the stock price $S_{i}$ includes the following individual steps:

$$
S_{5}-S_{0}=\left(S_{1}-S_{0}\right)+\left(S_{2}-S_{1}\right)+\left(S_{3}-S_{2}\right)+\left(S_{4}-S_{3}\right)+\left(S_{5}-S_{4}\right)
$$

and, in general:

$$
S_{N}-S_{0}=\sum_{i=1}^{N} d S_{i} d S_{i}=S_{i}-S_{i-1}
$$

Because stock price differences present absolute monetary amounts, according to this model $€ 1,000$ would have the same deviations as $€ 10$. Indeed, this is not the case in real life, which requires proportional deviations. Accordingly, the model has to be modified in such away that individual steps are not added, but multiplied as follows:

$$
\frac{S_{5}}{S_{0}}=\frac{S_{1}}{S_{0}} \frac{S_{2}}{S_{1}} \frac{S_{3}}{S_{2}} \frac{S_{4}}{S_{3}} \frac{S_{5}}{S_{4}} \text {, in general: } \frac{S_{N}}{S_{0}}=\prod \frac{S_{i}}{S_{i-1}}
$$

Following the logarithm characteristics, such a model is derived from the above and the final result is:

$$
d \ln \left(S_{i}\right)=\ln \left(S_{i}\right)-\ln \left(S_{i-1}\right)
$$


The logarithm is normally distributed, with variance equivalent to time. Therefore, the standard deviation is equivalent to the root of time.

$$
d \ln \left(S_{(t)}\right)=\sigma X \sqrt{d t}
$$

where $X=$ a random number drawn from a standardized normal distribution.

The random walk is generated through the random number $X$, with the mean 0 and the variance $\sigma \sqrt{d t}$. Based on the Central Limit Theory, the sum of independent random numbers, where each number does not significantly influence the sum, forms the normal distribution (Deutsch 1998). The expression $X \sqrt{d t}$ is known as a Wiener process and describes Brownian molecular motion in physics.

Finally, when the random walk is generated for the stock price with the expected return, the independent variable drift is added. The mean in this case is $\mu t$.

$$
d \ln \left(S_{(t)}\right)=\mu d t+\sigma X \sqrt{d t}
$$

For the creation of continuous numbers, Ito's lemma from stochastic analysis has been used and results in the following (Hull 2003):

$$
\begin{aligned}
& d S(t)=S(t)\left(\mu+\frac{\sigma^{2}}{2}\right) d t+S(t) \sigma X \sqrt{d t} \\
& S(t+d t)=S(t) X e^{\left(\mu-\frac{\sigma^{2}}{2}\right) d t+S(t) \sigma X \sqrt{d t}}
\end{aligned}
$$

Simulation of stock-price patterns was done using an Excel sheet. The simplest way is to use an Excel function for creating normally distributed random numbers: NORMINV(RAND(),0,1). A program in Visual Basic was developed in order to repeat the entire process many times.

Non-risky asset: Numerous interest rate models have been developed. Interest rate models are exceptionally important because they are used to estimate the term structure of interest rates of a single national economy, as well as to simulate interest rate movements. Models for simulating interest rates are equilibrium models of Vasicek (1977) and Cox, Ingersoll, and Ross (1985), the multifactor models of Brennan and Schwartz (1979) and Longstaff and Schwartz (1992), the two-factor model of Chen and Ross (2003), the "no-arbitrage" models of Ho-Lee (1986), and Hull and White (1990), nonstationary models, and others (Hull 2003).

This paper uses the Cox-Ingersoll-Ross (CIR) model, which was one of the first interest rate models. This model was chosen because interest rates cannot become negative, which is the case in the Vasicek model.

In 2003, the CIR model was used at most insurance companies in Germany (Fischer et al. 2003). Today, financial institutions use a one-factor Hull-White and Black-Karasinski model as Kaiser from German Association of Actuaries reported. These models however, include parameters from financial derivatives markets. Because derivatives are not traded in regional financial markets, it was decided not to use these models. The CIR model usually begins with assumptions about economic 
variables and derives the process for the short rate. The short rate $r$ at the time is the rate that applies to the short period of time at time $t$. It is also known as the instantaneous short rate. The real world is not relevant for the process for $r$, but the risk-neutral world. Ina risk-neutral world, in a very short period of time between $t$ and $t$ $+\delta t$, investors earn on average a return of $r(t) \delta t$ (Hull 2003).

In a one-factor equilibrium model, the process for $r$ involves only one source of uncertainty. Usually, the risk-neutral process for the short rate is described by Ito's process of the form:

$$
d r=m(r) d t+s(r) d z
$$

The instantaneous drift $m$ and instantaneous standard deviations are assumed to be functions of $r$, but are independent of time. In the CIR model, the risk neutral process for $r$ is:

$$
d r=a(b-r) d t+\sigma \sqrt{r} d z(H u l l 2003, p .542)
$$

Here, $r$ follows geometric Brownian motion. $a, b$, and $\sigma$ are constants.

An important difference between stock prices and bond prices is the phenomenon known as mean reversion. This model incorporates mean reversion, which means that interest rates pull back to some long-run average over time (Hull, 2003). The rate is pulled to level $b$ at rate $a$. Superimposed on this "pull" is the normally distributed stochastic term odz (Hull 2003, p.539).

Despite the name and purpose of the short-term interest rate models, these models are also used for simulating long-term rates. Using these models for long-term interest rates is criticized, and it was proven that their application may be incorrect in certain time periods. Deelstra (2000) has developed an advanced version of the CIR model for long-term interest rates. The disadvantage of the model is the high complexity of calculations, which makes it inappropriate for implementation in practice.

Step 3. Estimation of model parameters will be described for both risky and non-risky asset.

Risky asset: For a risky asset, a simple method of parameter estimation was used. By using sample statistics, daily continuous returns were calculated for daily discrete data. First, mean return and standard deviation were calculated for daily data. Second, daily data were annualized: the average daily return was multiplied by 250 (the number of trading days in a year), and the daily standard deviation was divided by $\sqrt{250}$ (Bruns et al. 2003). More sophisticated estimation methods for stock price stochastic models exist, but in this research focus is placed on the non-risky parameter.

Non-risky asset: The CIR model parameters for simulating interest rates are mean return $(\mu)$, standard deviation $(\sigma)$, and interest rate drift $(a)$. Mean return and standard deviation could be estimated by using sample statistics as shown for equities. However, it has been proven that such estimation does not adequately estimate interest rate movements. The focus of this research is on interest rate modeling, and therefore an advanced estimation approach is taken.

Fischer, May, and Walther $(2003,2004)$ described several manners of data calibration for CIR models. This was done due to the importance of parameter estimation, especially for actuaries, who use this model in practice. They found that while working with interest rate models, practitioners often used parameters estimated by experts that do not fit their real data or portfolio and may yield incorrect results. Thus, a parameter calibration should be prepared. 
Methods used for CIR model estimation include the method of least squares, maximum likelihood method, Kalman filter, and so on. For parameter estimation daily, weekly, or monthly bond return time series are needed. Data provided by the German Bundesbank are in discrete values, which is rarely the case in developed financial markets. The data in developing markets are very often in discrete values.

The discrete data need to be converted into continuous values in order to estimate the continuous model parameters. The covariance equivalent discretization method was developed by Deelstra and Parker (1995). The method uses the method of least squares and was developed in Excel as part of this paper.

Covariance equivalent discretization:

$$
r_{t}=\phi r_{t-1+} \sigma_{a} \sqrt[a]{\frac{2 \phi}{1+\phi} r_{t-1} \gamma a_{t}} \text { for } t=1,2,3
$$

where $\phi$ is a discrete drift of the interest rate,

is the long-term average of the interest rate, and $\sigma$ is the standard deviation

Implementation steps are following. Times series of interest rates are selected. A centered CIR model is created by subtracting the long-term interest rate from the interest rate for each period given:

$$
r_{t}=-r_{t}^{*}-\gamma
$$

where the centered CIR model is following: $d r_{+}=-k r_{+} d t+\sigma \sqrt{r_{t}-\gamma} d B_{t}$

The method of least squares is used to determine the parameters of discrete values as follows. For each value in the time series of interest rates, the residual value is calculated as follows:

Covariance equivalent discretization:

$$
\sigma^{2} a^{2}=\frac{\left(r_{t}-\phi r_{t-1}\right)^{2}}{\frac{2 \phi}{\phi-1}+\gamma}
$$

where $\phi$ is discrete interest rate drift.

Afterwards, the sum of residual values is calculated as RSS $=\sum_{i=1}^{N} \sigma_{a} a_{t}$, and by using the solver function in Excel, the RSS is minimized by changing the value of drift $\phi$.

Discrete volatility $\sigma=\sqrt{\frac{R S S}{N-1}}$ :, where $N$ is number of residual periods.

The conversion of volatility and interest rate drift from discrete to continuous values is calculated as following:

$$
\begin{aligned}
& \text { Continuous drift k: } \phi=e^{-k} \\
& \text { Continuous volatility } \sigma: \sigma_{a}^{2}=\sigma^{2} \frac{1-\phi^{2}}{2 k}=\sigma^{2} \frac{1-e^{-2 k}}{2 k}
\end{aligned}
$$

Step 4: Setup of simulations

The scenario definitions and results of parameter estimation are the following. 
Bull Markets: The risk-free interest rate is the overnight rate at the Frankfurt stock exchange, which amounted to $r_{F}=3.37 \%$ in this period. The following scenarios were created for bull markets (Table 1):

- The risky asset is the SASX 10 index.

- The risky asset is the gold price in EUR.

- The mean return for gold, hypothetical assumption for low standard deviation of $10 \%$.

Table 1

Scenario 1: Bull markets

\begin{tabular}{|c|c|c|}
\hline Time period & January 4th, 2006 & August 4th, 2008 \\
\hline \multicolumn{3}{|l|}{ SASX 10 index } \\
\hline Description & Daily return & Annual return \\
\hline Mean return & $0.05 \%$ & $12.65 \%$ \\
\hline St. Dev. & $1.78 \%$ & $28.20 \%$ \\
\hline \multicolumn{3}{|l|}{ GDBR 10 index } \\
\hline Parameter & Description & Value \\
\hline$\Gamma$ & Long-term average & $4.05 \%$ \\
\hline A & Discrete drift & 0.9875 \\
\hline$\Sigma a$ & Discrete volatility & $0.19 \%$ \\
\hline K & Continuous drift & $1.26 \%$ \\
\hline$\Sigma$ & Continuous volatility & $0.19 \%$ \\
\hline ro & $\begin{array}{l}\text { Interest rate at } \\
\text { inception }\end{array}$ & $3.28 \%$ \\
\hline \multicolumn{3}{|c|}{ Price of gold expressed in EUR } \\
\hline Description & Daily return & Annual return \\
\hline Mean return & $0.04 \%$ & $10.66 \%$ \\
\hline $\begin{array}{l}\text { Standard } \\
\text { deviation }\end{array}$ & $1.09 \%$ & $17.29 \%$ \\
\hline
\end{tabular}

Source: Author's calculations and Sarajevo Stock Exchange, German Bundesbank, Bloomberg

Bear Markets: The risk-free interest rate is the overnight rate at the Frankfurt stock exchange, which amounted to $r_{F}=1.83 \%$ in this period. The following scenarios were created for bear markets (Table 2):

- The risky asset is the SASX 10 index.

- The risky asset is the SASX 10 index and hypothetical assumption for high standard deviation of $30 \%$.

- The risky asset is SASX 10 index and hypothetical assumption for low standard deviation of $10 \%$.

No-trend Markets: The risk-free interest rate is the overnight rate at the Frankfurt stock exchange, which amounted to $r_{F}=0.607 \%$ in this period. The following scenarios were created for no-trend markets (Table 3):

- 3.1. The risky asset is the SASX 10 index from the no-trend period.

- 3.2.The risky asset is the SASX 10 index and hypothetical assumption for high standard deviation of $30 \%$.

- The risky asset is the SASX 10 index and hypothetical assumption for low standard deviation of $8 \%$. 
Table 2

Scenario 2:Bear Markets

\begin{tabular}{|c|c|c|}
\hline Time period & January 4th, 2006 & December 31st, 2012 \\
\hline \multicolumn{3}{|l|}{ SASX 10 index } \\
\hline Description & Daily return & Annual return \\
\hline Mean return & $-0.04 \%$ & $-10.61 \%$ \\
\hline St. Dev. & $1.51 \%$ & $23.84 \%$ \\
\hline \multicolumn{3}{|c|}{ GDBR 10 index } \\
\hline Parameter & Description & Value \\
\hline$\Gamma$ & Long-term average & $3.17 \%$ \\
\hline A & Discrete drift & 1.0003 \\
\hline$\Sigma a$ & Discrete volatility & $0.31 \%$ \\
\hline K & Continuous drift & $-0.03 \%$ \\
\hline$\Sigma$ & Continuous volatility & $0.31 \%$ \\
\hline ro & $\begin{array}{l}\text { Interest rate at } \\
\text { inception }\end{array}$ & $3.33 \%$ \\
\hline \multicolumn{3}{|c|}{ Price of gold expressed in EUR } \\
\hline Description & Daily return & Annual return \\
\hline Mean return & $0.06 \%$ & $14.73 \%$ \\
\hline $\begin{array}{l}\text { Standard } \\
\text { deviation }\end{array}$ & $1.20 \%$ & $18.90 \%$ \\
\hline
\end{tabular}

Source: Author's calculations and Sarajevo Stock Exchange, German Bundesbank

Table 3

Scenario 3: No-trend Markets

\begin{tabular}{ccc} 
Time period & January 4th, 2006 & December 31st, 2012 \\
\hline SASX 10 index & Daily return & Annual return \\
\hline Description & $0.02 \%$ & $1.93 \%$ \\
\hline Mean return & $1.18 \%$ & $18.41 \%$ \\
\hline St. Dev. & \multicolumn{1}{c}{ Description } & Value \\
\hline GDBR 10 index & Long-term average & $2.94 \%$ \\
\hline $\begin{array}{c}\text { Parameter } \\
\Gamma\end{array}$ & Discrete drift & 1.000 \\
\hline$A$ & Discrete volatility & $0.30 \%$ \\
\hline$\Sigma a$ & Continuous drift & $-0.02 \%$ \\
\hline$K$ & Continuous volatility & $0.30 \%$ \\
\hline$\Sigma$ & Interest rate at & \\
\hline \multicolumn{2}{c}{ inception } & $3.02 \%$ \\
\hline
\end{tabular}

Price of gold expressed in EUR

\begin{tabular}{lll} 
Description & \multicolumn{1}{c}{ Daily return } & Annual return \\
Mean return & $0.07 \%$ & $17.47 \%$ \\
\hline $\begin{array}{l}\text { Standard } \\
\text { deviation }\end{array}$ & $1.07 \%$ & $16.85 \%$
\end{tabular}

Source: Author's calculations and Sarajevo Stock Exchange, German Bundesbank, Bloomberg

Three portfolios A, B, and C were built with initial portions of risky and risk-free assets as follows:

- Aggressive portfolio A with 50\% risky asset, $50 \%$ risk-free asset, and CPPI $m=5$.

- Moderate portfolio B with 30\% risky asset, 70\% risk-free asset, and CPPI $m=3$.

- Conservative portfolio $C$ with $20 \%$ risky asset, $80 \%$ risk-free asset, and CPPI $m=2$. 
Fees: Cesari and Cremonini (2003) calculated $0.3 \%$ fee on transaction volumes and as a correction to the option volatility according to Leland's (1985) formula, whereas Dichtl and Drobetz (2010) used a $0.1 \%$ transaction fee. In this research, the fee used for buy-and-hold and constant-mix strategy was $0.5 \%$ on transaction volume. It is assumed that lower business volumes will be executed, which usually require higher investment fees. The rebalancing in a constant-mix strategy is done on a monthly basis.

The fees in the CPPI investment strategy are usually $1.5 \%$ of the portfolio value (Constantinou et al. 2008, p.27). In this study, the calculation of fees was made based on the method used by one investment bank. This method calculates fees of $0.5 \%$ to $1 \%$ on yearly level of non-risky asset volume, and $1 \%$ on risky asset volume if the option of leverage is used (Bertsch et al. 2004). In this study, the leverage effect of the CPPI strategy is not tested. The fee amount is calculated on a daily level and is subtracted from the value of non-risky asset.

\section{Step 5: Evaluation of investment strategies}

Evaluation method: Monte Carlo simulations were repeated 5,000 times. For each scenario, an average result for the following risk/return measures has been calculated: average return, standard deviation, Sharpe ratio, Sortino ratio, and Return at Risk (RaR). The Sharpe ratio is equal to the portfolio excess return over the risk-free rate $r_{F}$ divided by portfolio standard deviation.

$$
\text { Sharpe ratio }=\frac{\text { meanreturn }-r_{F}}{\sigma}
$$

The Risk Free Rate $r_{F}$ : in the absence of the risk-free rate for the Bosnian capital market, the most appropriate approach would be to follow Damodoran's (2008) method. This method uses the risk-free rate from the market with the highest ranking and makes a correction for the difference in inflation. However, in the CPPI strategy in this paper, in addition to local equities, there is an investment in German government bonds, which is why the risk-free rate from the German bond market was taken directly without making any adjustments.

The Sortino ratio, introduced by Sortino and Price (1994), is the mean return divided by downside deviation.

$$
\text { Sortino ratio }=\frac{\text { meanreturn }}{\text { downsidedeviation }}
$$

Downside deviation is defined as the shortfall risk, or the risk that the result is below zero.

$$
\text { Downside deviation }=\sqrt{\sum_{r_{t}} \frac{r^{2}}{T}}
$$

In the case of negative mean returns, the benchmarks of the Sharpe and Sortino ratios might be misleading (higher values for higher risk values). Therefore, Return at Risk is preferred, which is defined as follows:

Return at Risk is similar to the mean variance utility function with the risk aversion parameter $Y$, as well as the Value at Risk approach in relative values, in which $Y$ presents the probability level.

$$
\text { Return at Risk, RaR = mean return }-\mathrm{y} \text { volatility }
$$

RaR may be defined as minimum return with $(1-h) \%$ probability

$$
P(r>\operatorname{RaR})=1-\text { hi RaR }=\text { mean return }+P^{-1}(h) \sigma
$$

where $P^{-1}$ is an inverse distribution function. The parameter $y$ is equal to 2.33 , which is $1-h=99 \%$ probability. 
The lower the RaR, the better the portfolio performance, whereas in the case of the Sharpe and Sortino ratio higher values mean better portfolio performance (Cesari et al. 2003)

\section{Results}

The results for each scenario of Monte Carlo simulations are shown in the Appendix. Full set of simulation documents are available from the author upon request. In Table 4 , the best strategy is indicated for each market condition.

Table 4

The Best Strategy depending on Market Conditions and Benchmarks

\begin{tabular}{|c|c|c|c|}
\hline \multicolumn{4}{|c|}{ Average Return } \\
\hline & High volatility & Low volatility & Medium volatility \\
\hline Bull & $\mathrm{BH} \mathrm{A}$ & $\mathrm{CPPI} m=5$ & CPPI $m=5$ \\
\hline Bear & CPPI $m=2$ & CPPI $m=2$ & CPPI $m=2$ \\
\hline No trend & $\mathrm{BH} \mathrm{B}$ & $\mathrm{CPPI} m=2$ & CPPI $m=2$ \\
\hline \multicolumn{4}{|l|}{ St. Dev. } \\
\hline & High volatility & Low volatility & Medium volatility \\
\hline Bull & CMC & CMC & CMC \\
\hline Bear & CPPI $m=2$ & $\mathrm{BH} C / \mathrm{CPPI} m=2$ & $\mathrm{BHC}$ \\
\hline No trend & $\mathrm{BHC}$ & $\mathrm{BHC}$ & $\mathrm{BHC}$ \\
\hline \multicolumn{4}{|c|}{ Sharpe ratio } \\
\hline & High volatility & Low volatility & Medium volatility \\
\hline Bull & $C M B$ & $\mathrm{BH} \mathrm{A}$ & CMA \\
\hline Bear & CPPI $m=2$ & $\mathrm{CPPI} m=2$ & $\mathrm{BH} C / \mathrm{CPPI} m=2$ \\
\hline No trend & $\mathrm{BH} C$ & $\mathrm{CPPI} m=2$ & $\mathrm{BHC}$ \\
\hline \multicolumn{4}{|c|}{ Sortino ratio } \\
\hline & High volatility & Low volatility & Medium volatility \\
\hline Bull & CMC & CMC & CMC \\
\hline Bear & CPPI $m=2$ & $\mathrm{CPPI} m=2$ & $\mathrm{BH} \mathrm{C} / \mathrm{CPPI} \mathrm{m}=2$ \\
\hline No trend & $\mathrm{BH} C$ & $\mathrm{BH} \mathrm{C}$ & $\mathrm{BHC}$ \\
\hline \multicolumn{4}{|c|}{ Return at Risk } \\
\hline & High volatility & Low volatility & Medium volatility \\
\hline Bull & CMC & $\mathrm{CMC} / \mathrm{BH} \mathrm{A}$ & CMC \\
\hline Bear & CPPI $m=2$ & $\mathrm{BH} C / \mathrm{CPPI} m=2$ & $\mathrm{BH} \mathrm{C} / \mathrm{CPPI} \mathrm{m}=2$ \\
\hline No trend & $\mathrm{BHC}$ & $\mathrm{BH} C / \mathrm{CPPI} m=2$ & $\mathrm{BHC}$ \\
\hline
\end{tabular}

Source: Author

The main conclusions of the evaluations are following:

Average Return: According to average return, the CPPI strategy is superior in all market conditions, except for a bull market and no-trend markets with high volatility. The reason that it is the second-best strategy in bull and no-trend markets with high volatility is high transaction costs in times of great turbulence in the market. On the other hand, an aggressive buy-and-hold strategy fully profits from the rising risky-asset value without any transaction costs. In no-trend markets with low and medium volatility, constant-mix was slightly worse than CPPI and buy-and-hold.

In bull markets with different levels of volatility, the constant-mix strategy was the worst strategy according to average return because it reduces the share of risky asset when it rises and does not participate in the rising market values enough. In 
bear markets, the CPPI strategy was the absolute winner, offering capital protection even in times of high market volatility. Constant-mix was again the worst strategy in line with expectations.

Risk Ratios: According to Return at Risk ratio, in a bull market a conservative constant-mix strategy was the best. The second-best strategy was buy-and-hold, and CPPI was the worst, disregarding the level of risk taken and volatility level. In a bear market with high volatility, a conservative CPPI was the winning strategy, whereas in low and medium markets volatility-conservative CPPI and conservative buy-andhold had the same results. In no-trend markets, a conservative buy-and-hold strategy was the best strategy, the CPPI strategy was second, and constant-mix was the worst.

According to the Sortino and Sharpe ratio, a conservative constant-mix was the best in bull markets, and a conservative CPPI in bear markets. In no-trend markets, conservative buy-and-hold was the best.

\section{Conclusion}

The CPPI investment strategy is appropriate for use in SEE markets, and using it would contribute to the further development of financial markets and diversification of portfolio risks. The Monte Carlo simulation in which the data on bond returns have discrete values should be implemented by a covariance-equivalent discretization method. It was discovered that transaction costs play an important role in the strategy evaluation, and the results slightly differ from developed markets due to higher transaction costs.

The results of Monte Carlo simulations have shown that a conservative CPPI strategy is superior according to average return in all market conditions except for high volatility scenarios in bull and no trend markets, where the buy-and-hold strategy was better. Perold and Sharpe (1995) found that CPPI would be the best in bull and bear markets, and a constant-mix strategy in times of no-trend markets. This research proves that transaction costs play an important role in the outcome of the strategy; these costs were not taken into account in Perold and Sharpe's (1995) study. In their study, high volatility did not affect CPPI and constant-mix strategy results. The reason for the different result in no-trend markets may be explained by Perold and Sharpe's (1995) conclusion that the constant mix-strategy may be less successful if the markets for the risky asset end far from the starting point.

According to risk-adjusted measures, a conservative CPPI yielded the best results in bear markets, whereas a conservative constant-mix strategy had the best results in bull markets and conservative buy-and-hold in no-trend markets. The results in bear and bull markets are in line with Cesari and Cremonini's (2003) study. In no-trend markets, the winning strategy was CPPI according to Cesari and Cremonini, whereas in the case at hand buy-and-hold was better. This may be explained by higher transaction costs used in this study due to local market conditions. These conclusions contribute to the research on the CPPI strategy in emerging markets.

The CPPI strategy is an attractive option for investing in local markets due to the simplicity of its implementation and testing results. Based on Schottle and Werner's (2010) research which proves that the CPPI strategy is most successful when the risky component of the portfolio is optimized, a carefully selected and optimized portfolio of stocks in the SEE region would be the recommendation for the risky component of the portfolio. Non-risky assets of the portfolio cannot be taken from the regional markets because government bond markets in the region are not liquid enough. Non-risky assets should be taken from the German government bond market, which offers the highest degree of liquidity and safety. For those that are willing to accept 
more risk, the Polish market presents an alternative because it also offers a high degree of safety and liquidity.

The CIR model was used to simulate interest rates, but further studies should include more advanced models such as Hull-and-White and multifactor models, and test the effect on the CPPI investment strategy. In parallel, simulation of the risky component of the portfolio would need to be tested by using non-normal distribution of returns, including a jump-diffusion effect. This would test the CPPI strategy when both risky and non-risky assets are exposed to a greater level of uncertainty.

Further study should define the minimum and maximum trading volumes and trading frequency for successful portfolio insurance strategy implementation in SEE.

\section{References}

1. Bachelier, L. (1900), "Theorie de la speculaction", Annales scientifiques de l'Ecole Normale Superieure, Vol 17., pp. 21-86, translated as "Theory of Speculation" in Cootner, P.H., The Random Character of Stock Market Prices, Cambridge, MIT Press 1967

2. Banking Agency of Federation of Bosnia and Herzegovina, Information service www.fba.ba

3. Bassak, S. (2002), "Comparative study of portfolio insurance", Journal of Economic Dynamics \& Control, Vol. 26. No. 7-8, pp. 1217-1241

4. Belgrade Stock Exchange: www.belex.rs / (07. April 2012)

5. Bertsch, M./Pelliccioli, L./Tiers, A./Lenhart, M./Mastrangelo, J.F. (2004), JP Morgan Financial Engineering, Synthetic Portfolio Insurance - an Overview, 07.06.2004

6. Blattberg, R. C., Nicholas J. G. (1974), "A Comparison of the Stable and Student Distributions as Statistical Models for Stock Prices." Journal of Business, Vol. 47 No. 2, pp. 244-280.

7. Bodie, Z., Kane, A., Marcus, A.J. (2005). Investments, 6th International Edition, Singapore, McGraw-Hill,

8. Bossert, T., Burzin, C. (2002), „Dynamische Absicherung von Aktienportfolios Constant Proportion Portfolio Insurance", in Kleeberg, J.M., Rehkugler, H., Handbuch Portfoliomanagement, 2. Auflage, Bad Soden, pp. 129-157.

9. Bouchard, J.P. (2008), Models of Randomness and Complexity, from Turbulence to Stock-Markets, The Physics of Aestetics, Leonardo, Vol. 41 No 3, pp. 239-243

10. Bruns, C., Meyer-Bullerdiek F. (2003).Professioneles Portfolio Management: Aufbau, Umsetzung und Erfolgskontrolle strukturierter Anlagenstrategien, Stuttgart,Schäffer Poeschel Verlag

11. Cesari, R., Cremonini, D. (2003), "Benchmarking, portfolio insurance and technical analysis: a Monte Carlo Simulation of dynamic strategies of asset allocation", Journal of Economic Dynamics \& Control, Vol 27. No 6., pp. 987-1011

12. Constantinou, N., Khuman, A.D., Maringer, D. (2008), "Constant Proportion Portfolio Insurance: Statistical Properties and Practical Implications", Working Paper No. 023-08, University of Essex, August 2008

13. Cottrell, M, de Bodt, E., Gregoire, Ph. (1996),"Simulating interest rate structure evolution on a long term horizon: a Kohonen map application". Proceedings of Neural Networks in the Capital Markets, Californian Institute of Technology, Passadena, World Scientific Ed., pp.162-175

14. Cox, J.C., Ingersoll, J.E., Ross, S. A. (1985), "A Theory of the Term Structure of Interest Rates", Econometrica, Vol. 53. No 2., pp.385-407.

15. Croatian National Bank, (2010), Yearly Report

16. Damodaran, A. (2008), „What is the riskfree rate? A Search for the Basic Building Block", Stern School of Business, New York University, available at: 
http://people.stern.nyu.edu/adamodar/pdfiles/papers/riskfreerate.pdf / 08. January 2012)

17. Deelstra, G. \& Parker, G. (1995), A Covariance Equivalent Discretisation of the. CIR Model. Proceedings of the 5th AFIR International Colloquium (Actuarial approach for Financing Risks), Brussels

18. Deelstra, G. (2000), "Long-term returns in stochastic interest rate models: applications", ASTIN Bulletin, The Journal of International Actuarial Association, Vol 30. No 1., pp. 123-140

19. Dersch, D. (2010), "Dynamic Portfolio Insurance without options" in Kiesel R., Schrerer M., Zagst R. Alternative investments and strategies, Singapure, World Scientific Publishing Co., pp. 201-224

20. Deutsch, H.P., Eller, R. (1998). Derivate und Interne Modelle, Modernes Risikomanagement, Stuttgart, Schäffer Poeschel Verlag

21. Dichtl, H., Drobetz, W. (2010),"On the popularity of CPPI strategy: A BehavioralFinance-Based Explanation and Design Recommendations", The Journal of Wealth Management, Vol.13 No. 2, pp.41-54

22. Fischer, T., May, A., Walther, B., (2003),,,Anpassung eines CIR-1-Modells zur simulation der Zinsstrukturkurve", (Estimation of CIR-1 Model for the Simulation of Interest Rate Curve), TU Darmstadt, Blätter der DGVFM (Official Journal of German Actuarial Society), pp. 193-206

23. Fischer, T., May, A., Walther, B., (2004),,Anpassung eines CIR-k-Modells zur simulation der Zinsstrukturkurve", (Estimation of CIR-k Model for the Simulation of Interest Rate Curve), TU Darmstadt, Blätter der DGVFM (Official Journal of German Actuarial Society), pp. 369-287

24. Fischer, T., Roehrl, A. (2003), "Risk and performance optimization for portfolios of bonds and stocks",Proceedings of the International AFIR Colloquium, Maastricht

25. German Bundesbank www.bundesbank.de / (08.April 2012)

26. Glassermann, P. (2003). Monte Carlo Methods in Financial Engineering, New York, Springer Verlag

27. Hull, J.C., (2003). Options, Futures and Other Derivatives, 5th International Edition, Upper Saddle River N.J., Prentice Hall

28. Kolb, R.W., (2003). Futures, Options and Swaps, Oxford, Blackwell

29. Kosowski, R., Neftci S. (2015). Principles of Financial Engineering, Third Edition, London, Academic Press

30. Laurent, A. (2003). Derivatives and the Asset Allocation Decision: a Synthesis between Portfolio Diversification and Portfolio insurance, Ph.D. diss., University of St.Gallen, Bamberg,Difo Druck

31. Leland, H.E., Rubinstein, M. (1988), ,The Evolution of Portfolio Insurance" in Luskin, D., Portfoliolnsurance: A Guide to Dynamic Hedging, New York, John Wiley \& Sons

32. Mandelbrot, B. (1963), "The Variation of Certain Speculative Prices" Journal of Business, Vol. 36 No. 4, pp. 394-419.

33. Mauboussin, M.J., Bartholdson, K. (2002),.,A Tail and two worlds, Fat tails and Investing" in The Consilient observer (1) 7, Credit Suisse First Boston, available athttp://www.trendfollowing.com/whitepaper/mauboussin.pdf //04. November 2011)

34. Meyer-Bullerdiek,F., Schulz,M. (2004). Dynamische Portfolio Insurance-Strategien ohne Derivate im Rahmen der privaten Vermögensverwaltung: eine theoretische und empirische Analyse, Frankfurt a. M., Lang Verlag

35. Meyer-Bullerdiek,F.M. (2003), „Portfoliolnsurance - StrategienimVergleich“, (ComparisonofPortfoliolnsuranceStrategies), DieBank, 01.08.2003, pp. 24-27 
36. Pain, D., Rand, J., (2008), "Recent Developments in Portfolio Insurance", Bank of England Quarterly Bulletin, Vol 48.No 1., pp.37 - 46

37. Patton, A. J. (2004), "On the Out-of-Sample Importance of Skewness and Asymmetric Dependence for Asset Allocation" Journal of Financial Econometrics, Vol. 2 No. 1, pp.130-168.

38. Perold, A.F., Sharpe, W.F. (1995), "Dynamic Strategies for Asset Allocation", Financial Analysts Journal, Vol 51., pp.149-160

39. Rudolf, M. (1994). Algorithms for Portfolio Optimization and Portfolio Insurance, Ph.D. diss., St.Gallen University, Verlag Paul Haupt Bern Stuttgart Vienna

40. Sarajevo Stock Exchange www.sase.ba / (10. April 2012)

41. Schöttle, K., Werner R. (2010), "On the benefits of robust asset allocation for CPPI strategies", in Kiesel, R., Schrerer, M., Zagst, R., Alternative investments and strategies, Singapure, World Scientific Publishing Co., pp. 295-328

42. Steiner, M., Bruns C. (2002). Wertpapiermanagement, 3. Auflage, Stuttgart, Schäffer Poeschel Verlag

43. Wozniak L. (2006), "What is the hottest derivatives product this year? CPPI structures" available at http://www.financeasia.com/News/35205, whats-thehottest-derivatives-product-this-year-cppi-structures.aspx / (08. June 2012)

44. www.ublfunds.com.pk, (2014), UBL Fund Managers Limited, Pioneers of CPPI based funds in Pakistan - Now introduce AIPPF-V, available at: http://www.ublfunds.com.pk/individual/2014/12/10/ubl-fund-managers-limitedpioneers-of-cppi-based-funds-in-pakistan-now-introduce-aippf-v / (25. august 2015)

45. Zagreb Stock Exchange: www.zse.hr / (06. April 2012)

46. Zaimovic, A. (2010). Assumptions and possibilities for application of estimation models for risk and returns in capital market of Bosnia and Herzegovina, unpublished Ph.D. diss., University of Sarajevo

47. Zhao, Y. (2000). Dynamic investment models with downside risk control, PhD diss. at the University of British Columbia (Canada) 


\section{Appendix}

Table 1

Simulation: bull market, high volatility

\begin{tabular}{lccccc}
\hline Investment Strategy & $\boldsymbol{\mu}$ & $\boldsymbol{\Sigma}$ & $\begin{array}{c}\text { Sharpe } \\
\text { ratio }\end{array}$ & Sortino ratio & RaR \\
\hline Constant-Mix A & $8,24 \%$ & $13,93 \%$ & 0,319 & 0,770 & $-0,242$ \\
\hline Buy-and-Hold A & $9,09 \%$ & $15,87 \%$ & 0,282 & 0,663 & $-0,279$ \\
\hline CPPI m = 5 & $8,59 \%$ & $17,52 \%$ & 0,077 & 0,513 & $-0,322$ \\
\hline Constant-Mix B & $6,41 \%$ & $8,44 \%$ & 0,329 & 0,996 & $-0,133$ \\
\hline Buy-and-Hold B & $7,26 \%$ & $10,35 \%$ & 0,273 & 0,819 & $-0,169$ \\
\hline CPPI m = 3 & $8,20 \%$ & $15,10 \%$ & 0,122 & 0,608 & $-0,270$ \\
\hline Constant-Mix C & $5,37 \%$ & $5,74 \%$ & 0,319 & 1,244 & $-0,080$ \\
\hline Buy-and-Hold C & $6,14 \%$ & $7,35 \%$ & 0,264 & 1,008 & $-0,110$ \\
\hline CPPI m = 2 & $7,18 \%$ & $11,04 \%$ & 0,176 & 0,777 & $-0,186$ \\
\hline SOUC: Author & & & & & \\
\hline
\end{tabular}

Source: Author

Table2

Simulation: bull market, medium volatility

\begin{tabular}{lccccc} 
Investment Strategy & $\boldsymbol{\mu}$ & $\boldsymbol{\Sigma}$ & $\begin{array}{c}\text { Sharpe } \\
\text { ratio }\end{array}$ & Sortino ratio & RaR \\
\hline Constant-Mix A & $6,66 \%$ & $8,57 \%$ & 0,404 & 1,023 & $-0,133$ \\
\hline Buy-and-Hold A & $7,27 \%$ & $9,55 \%$ & 0,395 & 0,931 & $-0,150$ \\
\hline CPPI A & $8,04 \%$ & $12,53 \%$ & 0,278 & 0,739 & $-0,212$ \\
\hline Constant-Mix B & $5,31 \%$ & $5,25 \%$ & 0,389 & 1,352 & $-0,069$ \\
\hline Buy-and-Hold B & $5,86 \%$ & $6,12 \%$ & 0,379 & 1,190 & $-0,084$ \\
\hline CPPI B & $7,02 \%$ & $9,64 \%$ & 0,277 & 0,880 & $-0,154$ \\
\hline Constant-Mix C & $4,60 \%$ & $3,67 \%$ & 0,352 & 1,709 & $-0,039$ \\
\hline Buy-and-Hold C & $5,07 \%$ & $4,34 \%$ & 0,359 & 1,488 & $-0,050$ \\
\hline CPPI C & $5,83 \%$ & $6,24 \%$ & 0,312 & 1,178 & $-0,087$ \\
\hline SOUDC: Author & & & & & \\
\hline
\end{tabular}

Source: Author

Table6

Simulation: bull market, low volatility

\begin{tabular}{lccccc}
\hline Investment Strategy & $\boldsymbol{\mu}$ & $\boldsymbol{\sigma}$ & $\begin{array}{c}\text { Sharpe } \\
\text { ratio }\end{array}$ & Sortino ratio & RaR \\
\hline Constant-Mix A & & & & & \\
\hline Buy-and-Hold A & $\mathbf{6 , 5 0 \%}$ & $5,00 \%$ & 0,644 & 1,774 & $-0,052$ \\
\hline CPPI A & $7,14 \%$ & $5,58 \%$ & 0,673 & 1,674 & $-0,059$ \\
\hline Constant-Mix B & $8,95 \%$ & $8,48 \%$ & 0,623 & 1,351 & $-0,108$ \\
\hline Buy-and-Hold B & $5,16 \%$ & $3,18 \%$ & 0,579 & 2,275 & $-0,023$ \\
\hline CPPI B & $7,19 \%$ & $5,65 \%$ & 0,630 & 2,091 & $-0,028$ \\
\hline Constant-Mix C & $4,47 \%$ & $2,38 \%$ & 0,479 & 2,718 & $-0,067$ \\
\hline Buy-and-Hold C & $4,93 \%$ & $2,71 \%$ & 0,567 & 2,493 & $-0,014$ \\
\hline CPPI C & $5,66 \%$ & $3,63 \%$ & 0,588 & 2,097 & $-0,028$ \\
\hline SOUTC: Author & & & & &
\end{tabular}

Source: Author 
Table7

Simulation: bear market, high volatility

\begin{tabular}{lccccc}
\hline Investment Strategy & $\boldsymbol{\mu}$ & $\boldsymbol{\sigma}$ & $\begin{array}{c}\text { Sharpe } \\
\text { ratio }\end{array}$ & Sortino ratio & RaR \\
\hline Constant-Mix A & $-2,77 \%$ & $14,77 \%$ & $-0,434$ & $-0,204$ & $-0,372$ \\
\hline Buy-and-Hold A & $-1,86 \%$ & $12,88 \%$ & $-0,377$ & $-0,238$ & $-0,319$ \\
\hline CPPI A & $1,93 \%$ & $4,61 \%$ & $-0,004$ & 0,747 & $-0,088$ \\
\hline Constant-Mix B & $-0,14 \%$ & $9,00 \%$ & $-0,238$ & $-0,002$ & $-0,211$ \\
\hline Buy-and-Hold B & $0,50 \%$ & $7,74 \%$ & $-0,264$ & 0,007 & $-0,175$ \\
\hline CPPI B & $2,54 \%$ & $3,53 \%$ & 0,211 & 1,194 & $-0,057$ \\
\hline Constant-Mix C & $1,03 \%$ & $6,22 \%$ & $-0,146$ & 0,220 & $-0,135$ \\
\hline Buy-and-Hold C & $1,52 \%$ & $5,42 \%$ & $-0,133$ & 0,294 & $-0,111$ \\
\hline CPPI C & $2,83 \%$ & $3,06 \%$ & 0,342 & 1,465 & $-0,043$ \\
\hline SOUC: AuthOr & & & & & \\
\hline
\end{tabular}

Source: Author

Table8

Simulation: bear market, medium volatility

\begin{tabular}{lccccc} 
Investment Strategy & $\boldsymbol{\mu}$ & $\boldsymbol{\sigma}$ & $\begin{array}{c}\text { Sharpe } \\
\text { ratio }\end{array}$ & Sortino ratio & RaR \\
\hline Constant-Mix A & $-3,20 \%$ & $11,47 \%$ & $-0,58$ & $-0,31$ & $-0,30$ \\
\hline Buy-and-Hold A & $-2,21 \%$ & $9,96 \%$ & $-0,47$ & $-0,31$ & $-0,25$ \\
\hline CPPI A & $0,56 \%$ & $6,88 \%$ & $-0,26$ & 0,10 & $-0,15$ \\
\hline Constant-Mix B & $-0,50 \%$ & $7,06 \%$ & $-0,34$ & $-0,07$ & $-0,17$ \\
\hline Buy-and-Hold B & $0,25 \%$ & $6,02 \%$ & $-0,32$ & 0,00 & $-0,14$ \\
\hline CPPI B & $0,91 \%$ & $5,60 \%$ & $-0,23$ & 0,19 & $-0,12$ \\
\hline Constant-Mix C & $0,76 \%$ & $4,98 \%$ & $-0,23$ & 0,20 & $-0,11$ \\
\hline Buy-and-Hold C & $1,34 \%$ & $4,33 \%$ & $-0,16$ & 0,35 & $-0,09$ \\
\hline CPPI C & $1,36 \%$ & $4,58 \%$ & $-0,16$ & 0,35 & $-0,09$ \\
\hline SOUC: Author & & & & & \\
\hline
\end{tabular}

Source: Author

Table9

Simulation: bear market, low volatility

\begin{tabular}{lccccc} 
Investment Strategy & $\boldsymbol{\mu}$ & $\boldsymbol{\sigma}$ & $\begin{array}{c}\text { Sharpe } \\
\text { ratio }\end{array}$ & Sortino ratio & RaR \\
\hline Constant-Mix A & $-3,72 \%$ & $5,07 \%$ & $-1,407$ & $-0,816$ & $-0,155$ \\
\hline Buy-and-Hold A & $-2,67 \%$ & $4,43 \%$ & $-1,036$ & $-0,694$ & $-0,130$ \\
\hline CPPI A & $0,37 \%$ & $3,13 \%$ & $-0,482$ & 0,157 & $-0,069$ \\
\hline Constant-Mix B & $-0,94 \%$ & $3,43 \%$ & $-0,814$ & $-0,311$ & $-0,089$ \\
\hline Buy-and-Hold B & $-0,06 \%$ & $3,05 \%$ & $-0,634$ & $-0,033$ & $-0,072$ \\
\hline CPPI B & $0,75 \%$ & $2,81 \%$ & $-0,395$ & 0,343 & $-0,058$ \\
\hline Constant-Mix C & $0,43 \%$ & $2,81 \%$ & $-0,502$ & 0,201 & $-0,061$ \\
\hline Buy-and-Hold C & $1,12 \%$ & $2,64 \%$ & $-0,275$ & 0,552 & $-0,050$ \\
\hline CPPI C & $1,19 \%$ & $2,64 \%$ & $-0,248$ & 0,588 & $-0,050$ \\
\hline SOUC: Author & & & & & \\
\hline
\end{tabular}

Source: Author 
Table 10

Simulation: no trend, high volatility

\begin{tabular}{lccccc}
\hline Investment Strategy & $\boldsymbol{\mu}$ & $\boldsymbol{\sigma}$ & $\begin{array}{c}\text { Sharpe } \\
\text { ratio }\end{array}$ & Sortino ratio & RaR \\
\hline Constant-Mix A & $3,39 \%$ & $14,82 \%$ & 0,029 & 0,300 & $-0,311$ \\
\hline Buy-and-Hold A & $3,50 \%$ & $15,10 \%$ & 0,162 & 0,220 & $-0,317$ \\
\hline CPPI A & $3,15 \%$ & $12,38 \%$ & 0,119 & 0,232 & $-0,257$ \\
\hline Constant-Mix B & $3,19 \%$ & $11,26 \%$ & 0,232 & 0,376 & $-0,230$ \\
\hline Buy-and-Hold B & $3,56 \%$ & $9,47 \%$ & 0,266 & 0,386 & $-0,185$ \\
\hline CPPI B & $3,37 \%$ & $10,55 \%$ & 0,188 & 0,332 & $-0,212$ \\
\hline Constant-Mix C & $3,10 \%$ & $8,06 \%$ & 0,309 & 0,522 & $-0,157$ \\
\hline Buy-and-Hold C & $3,47 \%$ & $6,69 \%$ & 0,385 & 0,578 & $-0,121$ \\
\hline CPPI C & $3,51 \%$ & $7,96 \%$ & 0,304 & 0,500 & $-0,150$ \\
\hline SOUC: Author & & & & & \\
\hline
\end{tabular}

Source: Author

Table 11

Simulation: no trend, medium volatility

\begin{tabular}{lccccc}
\hline Investment Strategy & $\boldsymbol{\mu}$ & $\boldsymbol{\sigma}$ & $\begin{array}{c}\text { Sharpe } \\
\text { ratio }\end{array}$ & Sortino ratio & RaR \\
\hline Constant-Mix A & $2,73 \%$ & $9,15 \%$ & $-0,043$ & 0,390 & $-0,186$ \\
\hline Buy-and-Hold A & $2,88 \%$ & $9,30 \%$ & 0,228 & 0,343 & $-0,188$ \\
\hline CPPI A & $2,85 \%$ & $8,88 \%$ & 0,174 & 0,331 & $-0,178$ \\
\hline Constant-Mix B & $2,90 \%$ & $7,05 \%$ & 0,346 & 0,546 & $-0,135$ \\
\hline Buy-and-Hold B & $3,02 \%$ & $5,87 \%$ & 0,387 & 0,593 & $-0,107$ \\
\hline CPPI B & $3,03 \%$ & $6,76 \%$ & 0,298 & 0,511 & $-0,127$ \\
\hline Constant-Mix C & $2,91 \%$ & $5,20 \%$ & 0,462 & 0,760 & $-0,092$ \\
\hline Buy-and-Hold C & $3,04 \%$ & $4,29 \%$ & 0,549 & 0,859 & $-0,070$ \\
\hline CPPI C & $3,12 \%$ & $4,87 \%$ & 0,478 & 0,770 & $-0,082$ \\
\hline SOUC: Author & & & & & \\
\hline
\end{tabular}

Source: Author

Table 12

Simulation: no trend, low volatility

\begin{tabular}{lccccc}
\hline Investment Strategy & $\boldsymbol{\mu}$ & $\boldsymbol{\sigma}$ & $\begin{array}{c}\text { Sharpe } \\
\text { ratio }\end{array}$ & Sortino ratio & RaR \\
\hline Constant-Mix A & $2,36 \%$ & $4,14 \%$ & $-0,224$ & 0,742 & $-0,073$ \\
\hline Buy-and-Hold A & $2,49 \%$ & $4,20 \%$ & 0,444 & 0,731 & $-0,073$ \\
\hline CPPI A & $2,52 \%$ & $4,39 \%$ & 0,397 & 0,683 & $-0,077$ \\
\hline Constant-Mix B & $2,78 \%$ & $3,49 \%$ & 0,630 & 1,077 & $-0,054$ \\
\hline Buy-and-Hold B & $2,70 \%$ & $2,99 \%$ & 0,703 & 1,160 & $-0,043$ \\
\hline CPPI B & $2,76 \%$ & $3,22 \%$ & 0,653 & 1,077 & $-0,047$ \\
\hline Constant-Mix C & $2,83 \%$ & $2,93 \%$ & 0,765 & 1,337 & $-0,040$ \\
\hline Buy-and-Hold C & $2,80 \%$ & $2,59 \%$ & 0,851 & 1,453 & $-0,032$ \\
\hline CPPI C & $2,88 \%$ & $2,70 \%$ & 0,841 & 1,406 & $-0,034$ \\
\hline SOUC: Author & & & & & \\
\hline
\end{tabular}

Source: Author 


\section{About the author}

Elma AgićŠabeta completed her master's thesis with JP Morgan London/Frankfurt. Her MBA was obtained from the European School of Business, University of Reutlingen in Germany. Since 2002 she has held various leading positions in the accounting/controlling divisions of HVB bank, Unicredit Group and BBI bank in Sarajevo. She has been engaged as a lecturer at the Sarajevo School of Science and Technology and guest lecturer at the University of Sarajevo. In 2015 she received her PhD from the Faculty of Economics in Sarajevo. Her main research interests include financial markets, asset management, risk management, risk modeling, life insurance, and banking. The author can be contacted at elma.agic@gmail.com. 